\title{
ESTIMATIVA DA TEMPERATURA DE GLOBO NEGRO A PARTIR DA TEMPERATURA DE BULBO SECO
}

Paulo Giovanni de Abreu', Valéria Maria Nascimento Abreu², Luziane Franciscon ${ }^{3}$, Arlei Coldebella ${ }^{4}$, Adriana Garcia do Amaral $^{5}$

\section{RESUMO}

Uma desvantagem para se fazer o diagnóstico bioclimático de uma determinada região é a inexistência de medições da temperatura de globo negro (TGN) nas estações meteorológicas distribuídas ao longo do país. Dessa forma, propõemse equações para estimar a temperatura de globo negro a partir da temperatura de bulbo seco (TBS), para animais criados confinados e ao ar livre. Foram utilizados dados de TGN eTBS medidos dentro e fora dos aviários, analisados utilizando um modelo de regressão para predizer a TGN em função de TBS. Foram utilizados 5664 dados para análise da temperatura interna e 337 dados para a temperatura externa. As análises foram realizadas por meio do software estatístico R. Observou-se uma forte relação entre a temperatura de globo negro e a temperatura de bulbo seco. Dessa forma, para animais alojados em ambientes cobertos com temperatura de bulbo seco entre 0 e $35^{\circ} \mathrm{C}$, é possível estimar o TGN a partir da TBS. O modelo de regressão linear ajustado aos dados apresentou $\mathrm{R}^{2}$ de 0,9788 e coeficiente de correlação de 0,9893 . Nos dados de temperatura externa, a relação de TGN e TBS não é tão evidente. Esse fato ocorreu devido à forte influência da radiação solar no termômetro de globo negro. É possível estimar a TGN a partir da TBS para ambiente coberto e ao ar livre.

Palavras-chave: Índices, conforto térmico, calor radiante.

\section{ABSTRACT \\ ESTIMATING BLACK GLOBE TEMPERATURE FROM THE DRY BULB TEMPERATURE}

One disadvantage of bioclimatic diagnosis in certain region is non-availability of black globe temperatures (BGT) in the meteorological stations situated in different parts of the country. This study was done to propose a equation to estimate BGT from the dry bulb temperature (DBT), in confined and outdoors systems. Data of BGT and DBT were collected from inside and outside poultry sheds and analyzed using regression models to predict BGT from DBT data. A total of 5664 and 337 temperature readings were obtained from inside and outside poultry house, respectively. A statistical analysis was done with the use of statistical software R. A strong correlation was found between BGT and DBT. It was concluded that in chickens sheds with DBT between 0 and $35^{\circ} \mathrm{C}$, BGT can be estimated using DBT readings. Adjusted linear regression model had $\mathrm{R}^{2}$ of 0.9788 with correlation coefficient of 0.9893 . The relationship was not so evident for temperatures outside the measured readings, due to strong effect of the solar radiation on black globe thermometer. It is possible to estimate BGT from DBT readings obtained from internal or external environment.

Keywords: indexes, thermic confort, radiant heat load.

\section{Recebido para publicação em 05/04/2011. Aprovado em 23/05/2011.}

1- Engenheiro Agrícola, D.Sc., Pesquisador da Embrapa Suínos e Aves, Vila Tamanduá, Concórdia-SC, e-mail: pabreu@cnpsa.embrapa.br

2-Zootecnista, D.Sc., Pesquisadora da Embrapa Suínos e Aves, Vila Tamanduá, Concórdia-SC, e-mail: valeria@cnpsa.embrapa.br

3- Estatística, M.Sc., Analista da Embrapa Florestas, Curitiba-PR, e-mail: luziane@cnpf.com.br

4- Médico Veterinário, D.Sc., Pesquisador da Embrapa Suínos e Aves, Vila Tamanduá, Concórdia-SC, e-mail: arlei@cnpsa.embrapa.br

5- Engenheira Agrícola, M.Sc., Depto. de Engenharia Agrícola, UFV, Viçosa-MG, e-mail: adrianagamaral@gmail.com 


\section{INTRODUÇÃO}

Construir instalações adequadas ao clima que permitam a manutenção de temperatura, umidade relativa, velocidade do ar, em limites que proporcionam ambiente ideal no interior das instalações de acordo com as exigências dos animais, sem aumento dos custos de produção, tem sido grande desafio. O conhecimento das condições climáticas locais em comparação com as exigências dos animais é fundamental nesse processo (ABREU \& ABREU, 2005).

As variáveis do clima de uma região ditam os níveis necessários de controle artificial no sistema de manejo e, consequentemente, no custo econômico do manejo microambiental (ABREU \& ABREU, 2005). Entretanto, com o melhor desempenho animal e consequentemente maior produção de carne, alguns problemas têm surgido, tais como os cardíacos e pulmonares, que poderiam ser mitigados com o desenvolvimento de condições ambientais apropriadas às necessidades dos animais (MATIAS \& PATARRA, 1995). Em qualquer estudo de respostas fisiológicas dos animais ao ambiente, ou aos efeitos do ambiente na eficiência, conforto e bem-estar animal, está clara a necessidade de expressar numericamente $o$ ambiente que lhe concerne.

Se o ambiente térmico é específico, quatro fatores devem ser considerados: temperatura e umidadedo ar, velocidade do vento e o calor radiante recebido das superfícies vizinhas. Essas quatro variáveis podem ser expressas por meio de índices, facilitando assim a comparação de diferentes ambientes. Vários desses índices foram propostos. Buffington et al. (1981) propuseram o Índice de Temperatura de Globo e Umidade (ITGU), que incorpora os efeitos combinados de temperatura, umidade e velocidade do ar e radiação para avaliar o conforto e o desconforto dos animais em determinado ambiente. Esse é o índice que melhor caracteriza o ambiente térmico do animal. No entanto, para o cálculo desse índice, é necessário o valor da temperatura de globo negro, obtido de um termômetro situado no centro de uma esfera oca de cobre, com diâmetro de $15 \mathrm{~cm}$ e espessura de $0,5 \mathrm{~mm}$, pintada externamente com tinta preta fosca (ABREU, 1998). O ITGU é calculado pela equação:

$\mathrm{ITGU}=0,72(\mathrm{Tgn}+\mathrm{Tbu})+40,6$

em que

Tgn $=$ temperatura do globo negro $\mathrm{em}^{\circ} \mathrm{C}$; e

$\mathrm{Tbu}=$ temperatura de bulbo úmido $\mathrm{em}{ }^{\circ} \mathrm{C}$.

Outro índice que dependente da temperatura de globo negro é a carga térmica de radiação (CTR). A CTR, proposta por Esmay (1969), expressa a radiação total recebida de todos os espaços ou partes da vizinhança foi calculada pelas equações:

$\mathrm{CTR}=\sigma(\mathrm{TRM})^{4}$

em que

$\mathrm{CTR}=$ carga térmica de radiação $\mathrm{em} \mathrm{W} \mathrm{m}^{-2}$; $\mathrm{TRM}=$ temperatura radiante média em $\mathrm{K}$; e $\sigma=$ constante de Stefan Boltzmann, 5,67 $10^{-8} \mathrm{~W} \mathrm{~m}^{-2} \mathrm{~K}^{-4}$.

$\mathrm{TRM}=100\left\{2,51 \mathrm{v}^{1 / 2}(\mathrm{Tgn}-\mathrm{Tbs})+(\operatorname{Tgn} / 100) 4\right\} 1 / 4$

em que

$\mathrm{v}=$ velocidade do vento em $\mathrm{m} \mathrm{s}^{-1}$; e

Tbs = temperatura de bulbo seco K.

Uma desvantagem do uso do ITGU e da CTR para o diagnóstico bioclimático de uma determinada região é a inexistência de medições da temperatura de globo negro nas estações meteorológicas distribuídas ao longo do país (YANAGI JUNIOR, 2006). Na tentativa de caracterizar o ambiente térmico, têm sido adotados e propostos outros índices como o índice de temperatura e umidade (ITU), desenvolvido por Thom (1959) e Deshazer \& Beck (1988); o índice de temperatura, umidade e velocidade do ar (ITUV) para galinhas poedeiras e frangos de corte, desenvolvido por Yanagi Junior et al. (2001) e Tao \& Xin (2003); e o índice ambiental de produtividade para frangos de corte (IAPfc), desenvolvido por Medeiros (2001). Esses índices são mais fáceis de serem aplicados, uma vez que a temperatura de bulbo seco, umidade relativa do ar e velocidade do ar são fornecidas pelas estações 
meteorológicas e compõem diversos bancos de dados. Porém, esses índices não incorporam os efeitos da carga térmica de radiação. Dessa forma, propõem-se equações para estimar a temperatura de globo negro a partir da temperatura de bulbo seco, para animais criados confinados e ao ar livre.

\section{MATERIAL E MÉTODOS}

Foram utilizados dados de temperatura de globo negro (TGN) e temperatura de bulbo seco (TBS) medidos dentro e fora dos aviários e analisados utilizando um modelo de regressão para predizer a TGN em função de TBS. Esses dados são de três experimentos diferentes: a) um experimento de ventilação e cama; b) um de cortina e luz; e c) outro de piso, coletados ao longo de três anos na Embrapa Suínos e Aves em Concórdia - SC. Foram utilizados 5664 dados para análise da temperatura interna e 337 dados para a temperatura externa. As análises foram realizadas por meio do software estatístico R.

Os três experimentos foram conduzidos em aviários convencionais de $12 \mathrm{~m} \mathrm{x} 10 \mathrm{~m}$, com cobertura de telha de cimento amianto e pé direito de $3 \mathrm{~m}$, divididos internamente em quatro boxes, com 200 aves cada. No experimento com cortina e luz, as aves foram criadas em seis lotes; e no de ventilação e cama em quatro lotes, ambos com reutilização de cama. As lâmpadas utilizadas foram incandescentes com intensidade de 60 watts. Os tratamentos testados no experimento de cortina e luz foram dois tipos de cortina (amarela e azul) e dois programas de iluminação (quase contínuo $-23 \mathrm{~L}$ : $1 \mathrm{E}$ e Intermitente $-16 \mathrm{~L}$ : $2 \mathrm{E}$ : $1 \mathrm{~L}: 2 \mathrm{E}: 1 \mathrm{~L}: 2 \mathrm{E})$. Onde $\mathrm{E}=$ escuro e $\mathrm{L}=$ luz. Esses tratamentos foram distribuídos da seguinte maneira: Aviário 1: Tratamento 1 - Cortina Azul e luz intermitente; Aviário 2: Tratamento 2 - Cortina Amarela e luz Intermitente; Aviário 3: Tratamento 3 - Cortina Amarela e luz quase contínua, Aviário 4: e Tratamento 4 - Cortina Azul e quase contínua. Foram utilizados 19.200 machos da linhagem Ross, distribuídos em quatro repetições, em um delineamento inteiramente casualizado, em esquema fatorial $6 \times 2 \times 2$ (lotes, programas de luz, cortina).

Os tratamentos testados no experimento de ventilação e cama foram dois sistemas de ventilação (fixo e oscilante) com abrangência de $10 \mathrm{~m}$ de distância e dois tipos de material de cama (palhada de soja e casca de arroz). Os ventiladores eram acionados por termostato quando a temperatura ambiente atingia $25{ }^{\circ} \mathrm{C}$, tendo sido adaptados com potenciômetro e regulador de velocidade para o tamanho do aviário. Esses tratamentos foram distribuídos da seguinte maneira: Aviário 1 - Sistema de Ventilação Fixo, boxes 2 e 3 com palhada de soja (C1); boxes 1 e 4 com casca de arroz (C2); Aviário 2 - Sistema de Ventilação Oscilante, boxes 2 e 3 com palhada de soja (C1); boxes 1 e 4 com casca de arroz (C2); Aviário 3 - Sistema de Ventilação Oscilante, boxes 1 e 4 com palhada de soja (C1); boxes 2 e 3 com casca de arroz (C2); e Aviário 4 - Sistema de Ventilação Fixo, boxes 1 e 4 com palhada de soja (C1); boxes 2 e 3 com casca de arroz (C2). O terceiro experimento foi conduzido com o objetivo de avaliar as condições térmicas ambientais em aviários com piso de concreto e de chão batido. $\mathrm{O}$ experimento foi realizado em duas épocas e os tratamentos testados foram piso de concreto e piso de chão batido.

Nos três experimentos, foram coletadas no centro de cada boxe e no ambiente externo a temperatura de bulbo seco e a temperatura de globo negro. Para os experimentos de cortina e luz e piso, as coletas de TBS e TGN foram realizadas 2 vezes por semana de 0 a 24 horas, de 3 em 3 horas; para o experimento de ventilação e cama, as coletas foram realizadas em intervalos de $3 \mathrm{em} 3$ horas, de 8 as 18 horas, na quarta, quinta e sexta semana de vida das aves. Os dados da $1^{a}$, $2^{2}$ e $3^{\underline{a}}$ semana foram descartados pela interferência dos sistemas de aquecimento.

Com base nos dados coletados em cada horário, foi determinada a curva de regressão ajustada para predizer a temperatura de globo negro a partir da temperatura de bulbo seco, no ambiente interno e externo da instalação. Para a validação das equações, foram utilizados os dados de um quarto experimento com 260 dados de temperatura interna e 232 dados para temperatura externa. Esse experimento foi realizado em granja comercial de frangos de corte localizada no município de Juiz de Fora, MG, entre os dias 6 de fevereiro e 4 de março de 2009, do $21^{\circ}$ dia de vida das aves, até o 46ํㅡㄹ dia de vida das aves. 
A orientação do galpão é leste-oeste, $2,5 \mathrm{~m}$ de pé-direito, cobertura de telhas de cimento amianto, piso de concreto, cama de serragem reutilizada, cortinas laterais e forro de lona plástica nas cores azul e amarela, respectivamente. A partir desses dados, foram calculadas a acurácia e a precisão do modelo proposto para estimar TGN em função de TBS. A acurácia de medição reflete o grau de concordância entre o resultado de uma medição e um valor verdadeiro do mensurando, a qual foi calculada pela média da diferença entre o valor de TGN medido e o valor estimado pela equação. A precisão, por sua vez, reflete o grau de concordância entre os resultados de medições obtidos sob as mesmas condições, a qual foi calculada pelo desvio padrão da diferença entre o valor de TGN medido e o valor estimado pela equação. Foram calculados também os coeficientes de correlação para verificar a associação entre os valores de TGN do quarto experimento e os valores de TGN estimados pelo modelo.

\section{RESULTADOS E DISCUSSÃO}

Na Figura 1 mostra-se o gráfico de TGN e TBS para os dados de temperatura de bulbo seco interna do aviário e a reta ajustada pelo modelo de regressão. Observa-se uma forte relação entre as duas medidas. Dessa forma, para animais alojados em ambientes cobertos com temperatura de bulbo seco entre 0 e $35^{\circ} \mathrm{C}$, é possível estimar a TGN a partir da TBS. Dessa forma, o modelo de regressão linear ajustado aos dados apresentou $\mathrm{R}^{2}$ de 0,9788 , coeficiente de correlação de 0,9893 e erro de predição de 3,19\%. Yanagi Junior et al. (2000) estimaram o índice de temperatura de globo e umidade a partir da irradiância solar global entre 8:00 e 16:00 horas e encontraram valores de coeficientes de correlação 0,94 a 0,97 , que consideraram uma estimativa razoável dos valores observados para todos os horários, apresentando erro médio de $1,31 \%$.

A equação que permite estimar a TGN a partir da TBS para ambiente coberto corresponde a:

$\mathrm{TGN}=0,456+1,0335 \mathrm{TBS}$

em que

TGN $=$ Temperatura de globo negro $\mathrm{em}^{\circ} \mathrm{C}$; e TBS $=$ Temperatura de bulbo seco em ${ }^{\circ} \mathrm{C}$.

Na Figura 2 mostra-se os dados de temperatura de globo negro (TGN) e de bulbo seco (TBS) para o ambiente externo e a curva ajustada aos dados. Verifica-se que o modelo para o ambiente de fora da instalação é quadrático, diferindo do ambiente coberto, que apresentou modelo linear. Nos dados de temperatura externa, a relação de TGN e TBS não é tão forte. Essa menor precisão pode ser vista na Figura 2, que tem maior dispersão dos dados. Esse fato ocorreu por causa da forte

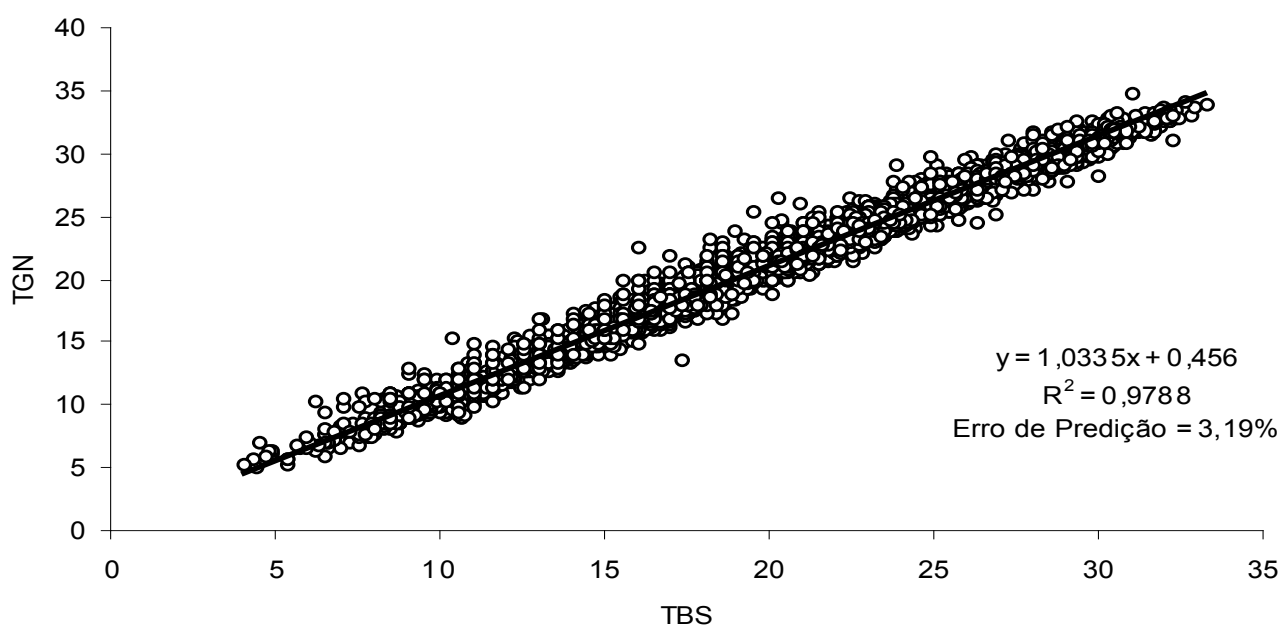

Figura 1. Dados de temperatura de globo negro (TGN) e de bulbo seco (TBS) para ambiente coberto e a reta ajustada pelo modelo de regressão. 


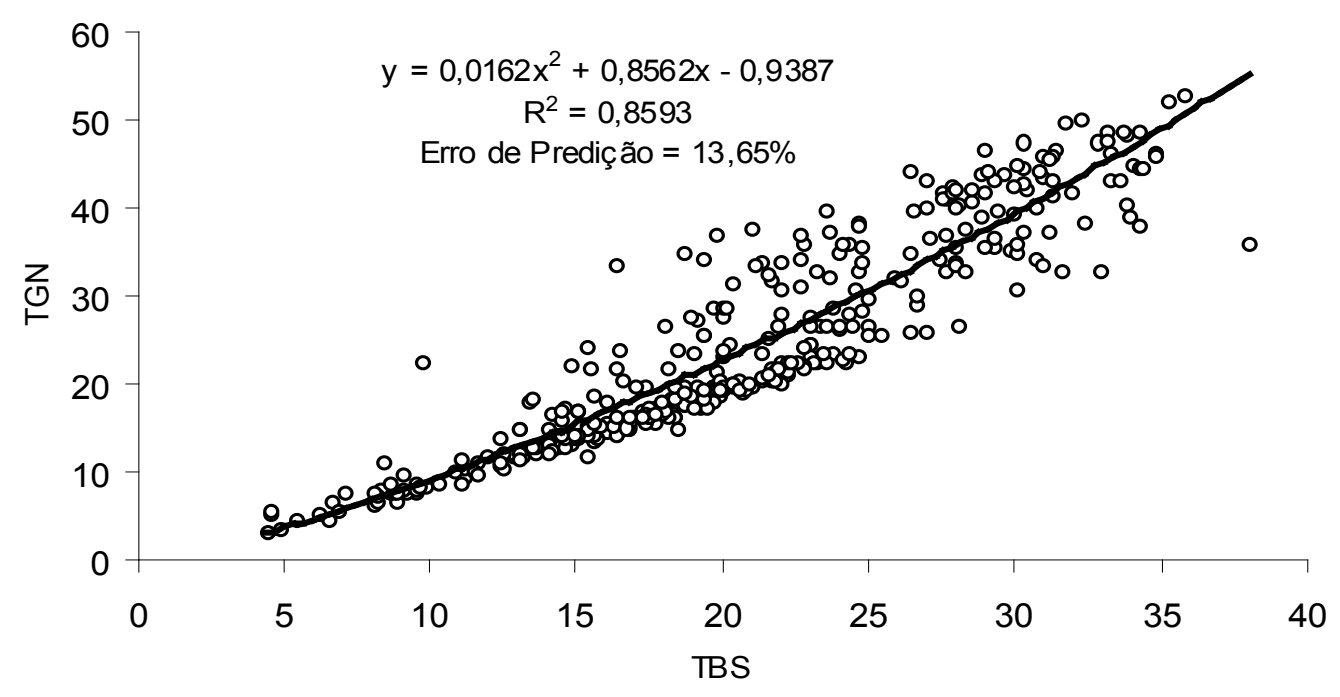

Figura 2. Dados de temperatura de globo negro (TGN) e de bulbo seco (TBS) para ambiente externo e a reta ajustada pelo modelo de regressão.

influência da radiação solar no termômetro de globo negro. Thorsson et al. (2007) avaliaram três métodos para estimar a temperatura média radiante em ambientes ao ar livre e verificaram que as discrepâncias ocorreram entre os métodos em função das rápidas mudanças na radiação, temperatura e velocidade do ar. Assim, a estimativa da TGN para o ambiente coberto foi mais precisa que a do ambiente externo por causa da menor variação das condições do ar interno.

O modelo de regressão quadrático ajustado aos dados apresentou $\mathrm{R}^{2}$ de 0,864 e erro de predição de $13,65 \%$.

A equação que permite estimar a TGN a partir da TBS para o ambiente externo corresponde a:

$\mathrm{TGN}=-0,9387+0,8562 \mathrm{TBS}+0,0162 \mathrm{TBS}^{2}$

em que

TGN $=$ Temperatura de globo negro $\mathrm{em}^{\circ} \mathrm{C}$; e TBS $=$ Temperatura de bulbo seco em ${ }^{\circ} \mathrm{C}$.

O coeficiente de correlação foi de 0,9648 para a temperatura interna e de 0,8485 para a temperatura externa e em ambas a associação é positiva e alta no experimento usado para validar as equações.

Para a temperatura interna, a acurácia foi de $-0,780{ }^{\circ} \mathrm{C}$ e a precisão de $0,755^{\circ} \mathrm{C}$. A temperatura externa apresentou valores de $-3,384{ }^{\circ} \mathrm{C}$ e 5,964
${ }^{\circ} \mathrm{C}$, respectivamente.

Turco et al. (2008) utilizaram dados de temperatura e radiação de estações meteorológicas para o desenvolvimento de modelos para estimar a TGN e encontraram para o período diurno coeficiente de correlação $(r=0,91)$ mostrando grande acurácia e desempenho do modelo para estimar os valores da TGN.

$\mathrm{Na}$ Figura 3 esta representada a relação entre os dados do experimento utilizado para validação e os dados estimados pelo modelo para a temperatura interna. Como pode ser observado, houve alta correlação entre os dados estimados e os dados medidos, mostrando que o modelo estimado pode ser utilizado de maneira confiável. No entanto, essa correlação não foi tão alta ao mostrar a relação entre os dados do experimento e os estimados pelo modelo para a temperatura externa (Figura 4).

\section{CONCLUSÃO}

- Nas condições da avaliação, em ambientes cobertos é possível e viável estimar a TGN a partir da TBS. Ao ar livre, apesar de ser possível estimar a TGN a partir da TBS, a viabilidade de aplicação depende da circunstância em que os dados serão utilizados por causa dos erros de predição elevados para essa condição. 


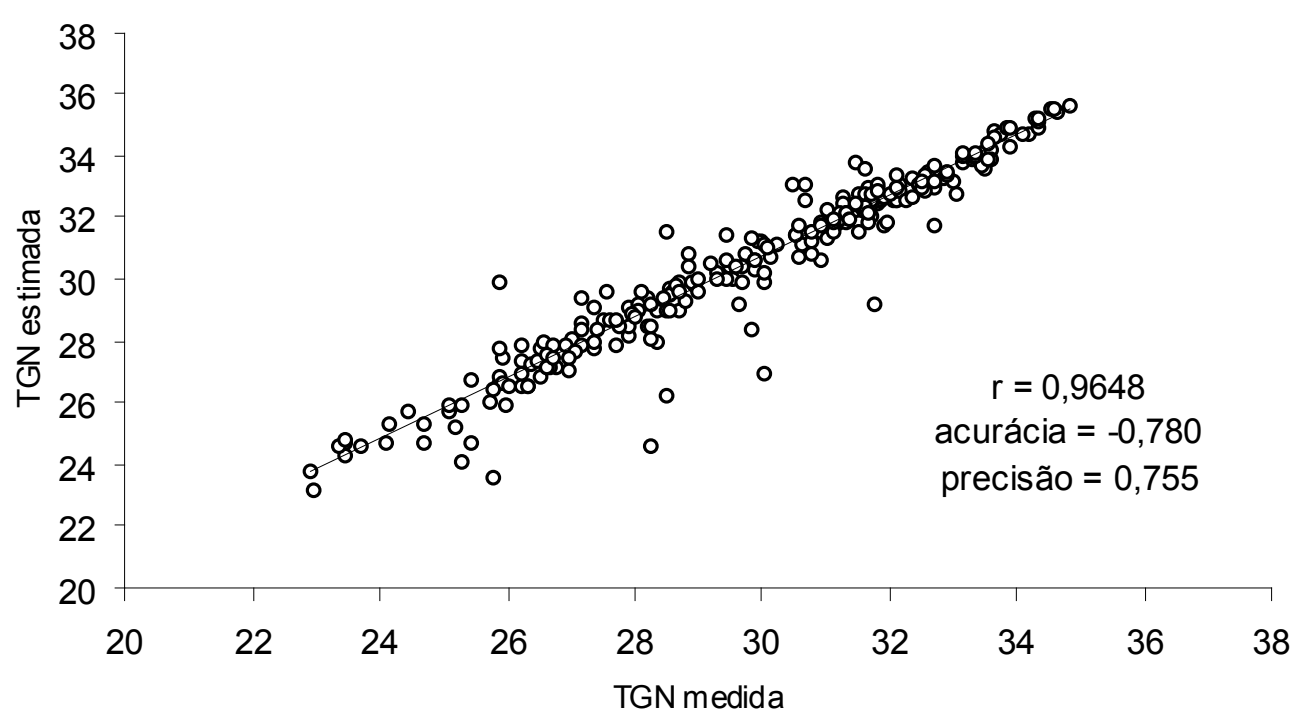

Figura 3. Dados de temperatura interna para o quarto experimento.

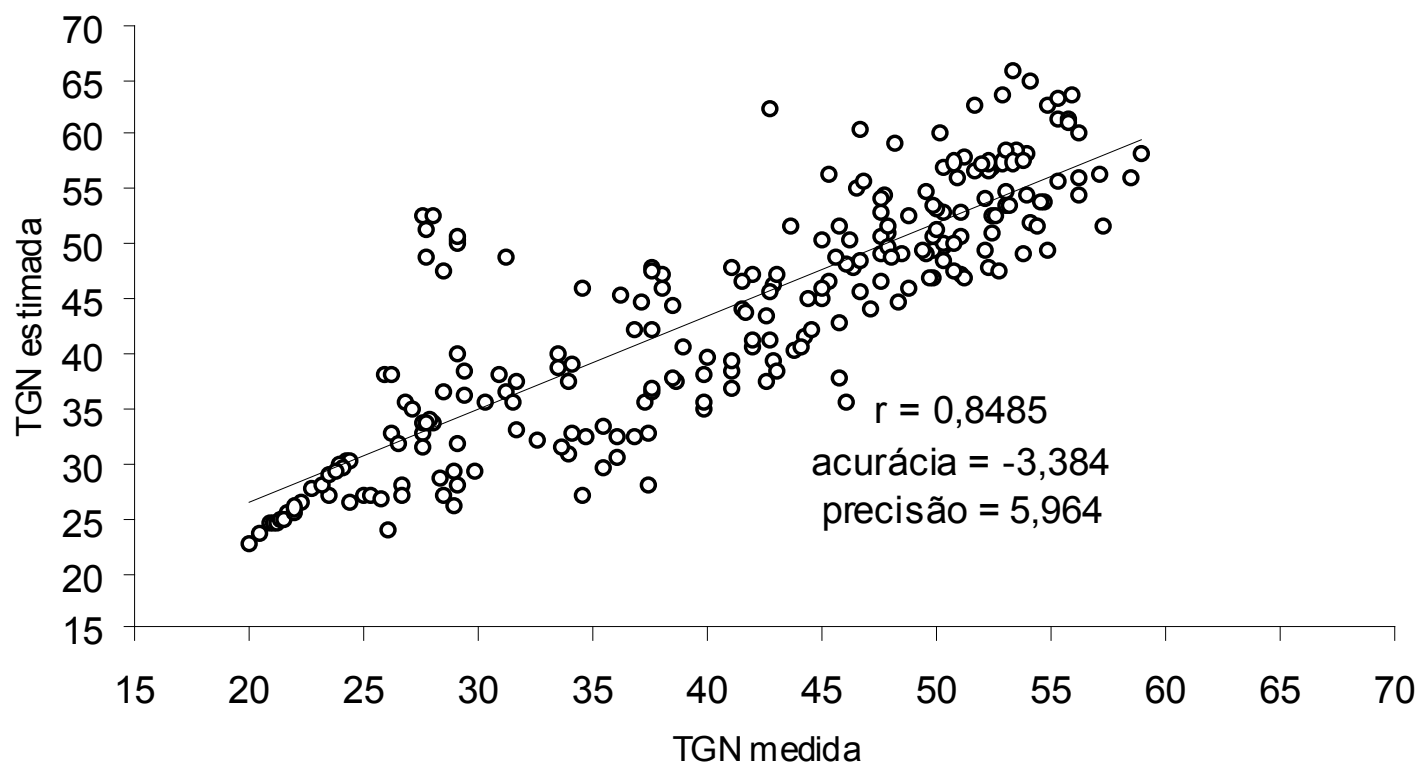

Figura 4. Dados de temperatura externa para o quarto experimento.

\section{REFERÊNCIAS BIBLIOGRÁFICAS}

ABREU, P.G.; ABREU, V.M.N. Diagnóstico bioclimático para produção de aves na mesorregião Pantanal Sul Mato-Grossense. In: CONFERÊNCIA APINCO 2005 DE CIÊNCIA E TECNOLOGIA AVÍCOLAS, 2005, Santos. Anais... Campinas: FACTA, 2005a. p.188.

ABREU, V.M.N.; ABREU, P.G. de. Diagnóstico bioclimático para produção de aves na mesorregião Nordeste Baiano. Concórdia: Embrapa Suínos e Aves, 2004. 5p. (Embrapa Suínos e Aves. Comunicado Técnico, 380).

ABREU, P.G. Sistemas de aquecimento em piso, com resistência elétrica, para criação de aves. Viçosa: UFV, 1994. 82p. Tese [Mestrado em Construções Rurais e Ambiência] - Universidade Federal de Viçosa, Viçosa, MG.BUFFINGTON, 
D.E. et al. Black globe-humidity index (BGHI) as comfort equation for dairy cows. Transactions of the ASAE, St. Joseph, v.24, n.3, p.711-714, 1981.

DESHAZER, J.A.; BECK, M.M. University of Nebraska Report for Northeast regional poultry project NE-127. Lincoln: Agricultural research Division, Univ. of Nebraska. 1988.

ESMAY, M.L. Principles of animal environment. Westport: CT Avi Publishing Co., 1969. 325p.

MATIAS, I.; PATARRA, P. Genética: a invasão dos pintos avós e sua fórmula secreta. Revista Avicultura Industrial, v.9, p.34-60, 1995.

MEDEIROS, C.M. Ajuste de modelos e determinação de índice térmico ambiental de produtividade para frangos de corte. 2001. 115p. Tese [Doutorado em Construções Rurais e Ambiência] - Universidade de Federal de Viçosa, Viçosa, MG

TAO, X.; XIN, H. Acute synergistic effects of air temperature, humidity, and velocity on homeostasis of market-size broilers. Transactions of the ASAE, St. Joseph, v.46, n.2, p.491-497, mar./abr, 2003.

THOM, E. C. The discomfort index. Weatherwise, Boston, v.12, n.1, p.57-60, 1959.

THORSSON, S. et al. Different methods for estimating the mean radiant temperature in an outdoor urban setting. Int. J. Climatol. v.27, p.1983-1993, 2007.

TURCO, S.H.N. et al. Estimating black globe temperature based on meterological data. In: INTERNATIONAL CONFERENCE OF AGRICULTURAL ENGINEERING; BRAZILIAN CONGRESS OF AGRICULTURAL ENGINEERING， 37.; INTERNATIONAL LIVESTOCK ENVIRONMENT SYMPOSIUM - ILES, 8., 2008, Foz do Iguaçu. Technology for all: sharing the knowledge for development: Proceedings... Foz do Iguaçu: SBEA, 2008. 1 CDROM.

YANAGI JUNIOR, T. Inovações tecnológicas na bioclimatologia animal visando a aumento da produção animal: relação bem-estar animal x clima. Infobibos: Informações Tecnológicas, 2006. Disponível em: http://www.infobibos.com/ Artigos/2006_2/ITBA/Index.ht. Acesso em: 14 jun. 2010.

YANAGI JUNIOR, T. et al. Desenvolvimento de um Software para simular o índice de temperatura do globo e umidade em galpões avícolas. In: REUNIÃO ANUAL DA SOCIEDADE BRASILEIRA DE ZOOTECNIA, 37., 2000. Viçosa, MG. Anais... Viçosa: SBZ, 2000. CDRom.

YANAGI JUNIOR T.; XIN, H.; GATES, R.S. Modeling partial surface evaporative cooling of chickens. ASAE paper. n. 01-3011. St Joseph, MI: ASAE, 2001. 